\title{
Caracterização hidrológica de uma bacia hidrográfica urbana no Ceará utilizando Sistema de Informações Geográficas - SIG
}

\author{
Hydrological characterization of an urban watershed in Ceará using Geographic Information \\ System - GIS
}

Caracterización hidrológica de una cuenca urbana en Ceará utilizando el Sistema de Información Geográfica - SIG

\section{Resumo}

O processo de impermeabilização nas cidades tem causado elevado escoamento superficial e, consequentemente, causando cheias pela inexistência e/ou precariedade dos sistemas de drenagem. Sendo assim, esta pesquisa objetiva realizar um estudo acerca do escoamento superficial em uma microbacia urbana no município de Barro. Para a delimitação da bacia utilizou-se de um MDE proveniente da imagem SRTM, obtida pelo projeto TOPODATA. Para a delimitação da bacia, elaboração dos mapas de curvas de nível, rede de drenagem e mapa de declividade. Verificou-se que a região apresenta problemas de drenagem em época chuvosa, porém, a bacia apresenta pouca tendência a enchentes. Mostrando que o problema ocasionado na região em estudo, não vem por meio da caracterização da microbacia e sim pela ausência de um sistema de drenagem no local, fazendo com que toda água precipitada sobre a microbacia escoe de forma superficial.

Palavras-chave: Infraestrutura; Hidrologia urbana; Drenagem superficial.

\begin{abstract}
The impermeabilization process in cities has caused high surface runoff and, consequently, causing floods due to the inexistence and/or precariousness of drainage systems. Thus, this research aims to conduct a study about the surface runoff in an urban watershed in the city of Barro. For the delimitation of the basin an MDE from SRTM image, obtained by the TOPODATA project, was used. For the delimitation of the basin, contour maps, drainage network and slope map were elaborated. It was verified that the region presents drainage problems in the rainy season, however, the basin presents little tendency to flood. Showing that the problem caused in the region under study, does not come
\end{abstract}


through the characterization of the watershed but by the absence of a drainage system in place, causing all water precipitated on the watershed to drain superficially.

Keywords: Infrastructure; Urban hydrology; Surface drainage.

\section{Resumen}

El proceso de impermeabilización de las ciudades ha provocado elevados escurrimientos superficiales y, en consecuencia, inundaciones por la inexistencia y/o precariedad de los sistemas de drenaje. Por ello, esta investigación tiene como objetivo realizar un estudio sobre el escurrimiento superficial en una cuenca urbana del municipio de Barro. Para la delimitación de la cuenca se utilizó un MDE a partir de la imagen SRTM, obtenida por el proyecto TOPODATA. Para la delimitación de la cuenca, elaboración de mapas de contorno, red de drenaje y mapa de taludes. Se encontró que la región presenta problemas de drenaje en época de lluvias, sin embargo, la cuenca tiene poca tendencia a inundarse. Demostrando que el problema ocasionado en la región en estudio, no viene por la caracterización de la microcuenca, sino por la ausencia de un sistema de drenaje en el lugar, ocasionando que toda el agua precipitada sobre la microcuenca se escurra de manera superficial.

Palabras clave: Infraestructura; Hidrología urbana; Drenaje superficial.

\section{Introdução}

De acordo com Targa et al. (2012), a busca de construir edificações vem crescendo cada vez mais. Esse fato tem provocado a ocupação não planejada de áreas como as margens dos cursos d'água. Esse rápido crescimento das cidades no Brasil impermeabilizou o solo, ocasionando a diminuição da capacidade de absorção de água (Barboza et al., 2020).

Para Zanandrea (2016), o crescimento desordenado das cidades traz várias problemáticas no que se refere a drenagem das bacias urbanas, especialmente no escoamento das águas e na morfologia do canal. Para o autor, essas demandas são ocasionadas pela forma como a ocupação urbana se desenvolve, geralmente sem planejamento ambiental e urbano, sem controle do uso do solo em consonância com a falta de acompanhamento do sistema de saneamento básico com o crescimento das cidades.

Maus et al. (2007) complementam ao afirmar que o processo de impermeabilização nas cidades tem causado elevado escoamento superficial e, consequentemente, causando cheias pela inexistência e/ou precariedade dos sistemas de drenagem. Tais efeitos combinados resultam em sobrecarga nos sistemas de drenagem que, quando não desempenham sua função de modo eficiente, podem resultar em inundações, enxurradas e alagamentos na área urbana (Tasca et al., 2017). Bispo e Levino (2011) corroboram ao afirmar que a impermeabilização das áreas urbanas vem impedir o processo de infiltração da água no solo, por consequente resultando em picos de cheia, reduzindo a recarga do lençol freático e diminuindo a disponibilidade de água nos períodos secos.

A área de interesse desse estudo apresenta-se como uma região com ocupação do solo indevida por se tratar de uma região de bacia e não haver plano habitacional contendo todas as medidas necessárias para o desenvolvimento da região, alta impermeabilização e, em áreas não impermeabilizadas, grande parte do solo visualmente aparenta estar compactado, além do assoreamento nos corpos hídricos presentes na Microbacia Urbana do Barro - CE.

Esse estudo de caso justifica-se pela necessidade de estudo da drenagem urbana local, visto que as áreas de escoamento da água no período de chuva encontram-se impermeabilizadas causando graves problemas para população, como fortes enchentes, processos erosivos ativos, que privam a população de transitar com seus veículos e até mesmo a impossibilidade de tráfego dos moradores e acesso às suas residências. Dessa forma, torna-se necessário um estudo para quantificar o escoamento superficial gerado na área em estudo, através de modelagem chuva-vazão, para definir medidas eficientes na solução dos problemas causados pela falta de drenagem urbana. 


\section{Caracterização da Área em Estudo}

O município de Barro está localizado no Cariri Centro-Sul (Souza \& Sousa, 2020), localizado aos $7^{\circ} 10^{\prime} 37^{\prime \prime}$ de latitude Sul e aos $38^{\circ} 46^{\prime} 55^{\prime \prime}$ de longitude Oeste de Greenwich (Figura 1), com área de 711, 25 km² e população estimada em 22.758 habitantes (IBGE, 2020).

Figura 1: Localização de Barro - Ceará.

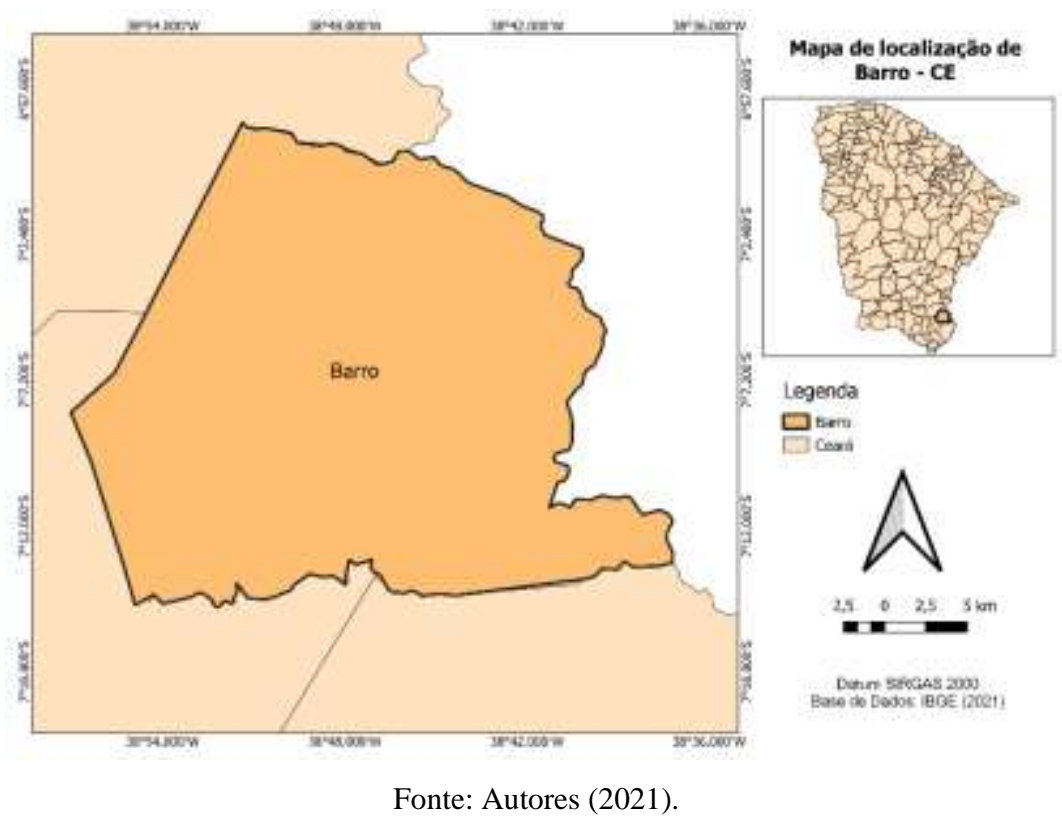

O município de Barro possui um clima tropical quente semiárido, com solo de características diversas, como: Neossolos Flúvicos, Neossolo Quartzarênico, Luvissolos, Neossolo Litólico e Argissolos. A geologia da região é composta por arenitos; arenitos e conglomerados; folhetos, siltitos e arenitos; micaxistos e metarritmitos. De acordo com Souza e Sousa (2020), o domínio hidrogeológico é composto por rochas sedimentares, cristalinas e depósitos aluvionares. Verificou-se que a predominância são os arenitos conglomerados, típico de fundo de rio, ou seja, região sedimentar e de deposição de areia.

Através dos dados obtidos no site do MAPBIOMAS, no qual fornece as imagens que possibilitam a análise detalhada da região, verificou-se que a área dessa microbacia se encontra predominantemente não vegetada com presença de algumas áreas de atividade agrícola. A microbacia hidrográfica urbana no município de Barro - CE, está localizada entre os bairros Jardim Raimundo Inácio e o Centro, tendo como eixo principal a rua João Tavares Neto.

\section{Procedimentos metodológicos}

\subsection{Delimitação automática da bacia hidrográfica em estudo}

Para a delimitação da bacia, elaboração dos mapas de curvas de nível, rede de drenagem e mapa de declividade foi utilizado um MDE que é oriundo da imagem SRTM, na qual foi possível obter por meio do projeto TOPODATA - INPE, o qual possui uma resolução espacial de 30 metros.

Para geração dos produtos cartográficos foi utilizado o software QGIS. Foi realizado os seguintes processos: a cena SRTM de altitude utilizada foi a da carta 07S39_ZN. Logo, foi projetada no sistema de coordenadas SIRGAS 2000, sistema esse adotado para o estudo em análise. A cena obtida através do INPE foi utilizada para que um mosaico fosse gerado. 
Posteriormente, tratou-se a imagem transformando-a em mosaico e passando-a do sistema de coordenadas geográficas para o sistema de coordenadas planas que é necessário para determinação da área e do perímetro, através da ferramenta raster > projeções > reprojetar coordenadas.

Para delimitação da bacia hidrográfica, também foi utilizado o programa QGIS, que de início, foi carregado o MDE na janela principal do referido software por meio da opção “Camada -> Adicionar Camada -> Raster. Na sequência, realizou-se o recorte da área que envolve a bacia tornando um polígono.

Inicialmente, verificou-se através da ferramenta Google Earth o ponto de exutório da bacia em análise para que fossem geradas, através do site TOPODATA, as cenas que contém os dados altimétricos da área de estudo em análise.

Para executar o processo, foi utilizado os algoritmos que o provedor GRASS fornece ao QGIS da Caixa de Ferramentas de Processamento através do menu Processes / Toolbox, utilizando o seguinte caminho: GRASS / Raster / r. watershed Orders ou digitalizamos r. watershed no mecanismo de busca. O parâmetro seguinte utilizado foi a introdução do tamanho mínimo de cada bacia fazendo um cálculo dos pixels do MDE.

Para este estudo, que está contida na cena 07S39_ZN, gerou-se o modelo digital de elevação (MDE), em que após inicializarmos a tarefa de projeto no Qgis, realizou-se a transferência do modelo raster MDT através da ferramenta v.to. rast. Attribute, em Input vector layer foi indicado o layer que contém as curvas de nível e posteriormente foi indicado em Name of column for 'attr' parameter a coluna que possui os valores das cotas. Na sequência, definiu-se as linhas de divisores de água por meio de análise altimétrica disposta na região.

\subsection{Morfometria}

Villela e Mattos (1975) afirmam que conhecer a fisiografia de uma bacia é extremamente importante pois através disso é possível avaliar o seu comportamento hidrológico, visto que ao se estabelecerem relações e comparações entre eles e dados hidrológicos conhecidos, é possível determinar o escoamento superficial nessa área. Logo, para conhecer a hidrologia de uma bacia hidrográfica, é preciso realizar a delimitação de sua área de influência e expressar os quantitativos dos parâmetros que se relacionam com a sua fisiografia e morfometria.

\subsubsection{Aspectos referentes a forma da bacia}

Esse tópico trata sobre a influência do formato da bacia hidrográfica na hidrologia, a saber que a forma interfere diretamente no comportamento hidrológico da bacia, a exemplo, o tempo de concentração e o escoamento superficial. (Villela \& Mattos, 1975)

\subsubsection{Coeficiente de Compacidade (KC)}

Coeficiente de Compacidade (KC) (equação 1) é a relação entre o perímetro da bacia e o perímetro de um círculo de mesma área que a bacia. Quanto maior o KC menor é a circularidade da bacia, maior o tempo de concentração e menor a tendência de ocorrer picos de enchente (Villela \&Mattos, 1975).

$$
K c=0,28 \frac{P}{\sqrt{A}}
$$

\subsubsection{Fator de forma (KF)}

Segundo Garcez e Alvarez (1988), Kf (Equação 2) é a relação entre a área de uma bacia e o quadrado da extensão do curso de água. 
$K f=\frac{A}{L^{2}}$

O Kf é fator de forma da bacia (adimensional); onde A é a área da bacia em $\left(\mathrm{km}^{2}\right)$ e $\mathrm{L}$ o comprimento do eixo principal em $(\mathrm{km})$. Quando uma bacia possui um Kf baixo indica que a mesma é menos sujeita a enchentes (VILLELA; MATTOS, 1975), conforme Quadro 1.

Quadro 1: Classificação quanto à forma.

\begin{tabular}{|c|c|}
\hline FATOR DE FORMA (KF) & Classificação \\
\hline $\mathrm{KF} \leq 0,5$ & TENDÊNCIA MENOR DE ENCHENTES \\
\hline $0,5<\mathrm{KF}<0,75$ & TENDÊNCIA MEDIANA DE ENCHENTES \\
\hline $\mathrm{KF}>0,75$ & TENDÊNCIA MAIOR DE ENCHENTES \\
\hline
\end{tabular}

Fonte: Goehr et al. (2015).

\subsubsection{Aspectos referentes a drenagem da bacia}

Através da densidade de drenagem é possível identificar a eficiência da drenagem de uma bacia hidrográfica. Os seus resultados são essenciais para afirmar se a drenagem dessa região é pobre, regular, boa ou muito boa (Teodoro et al., 2007).

\subsubsection{Densidade de Drenagem (DD)}

A densidade de drenagem (Equação 3) é a relação entre o comprimento total do canal (L) e a área da bacia, no qual se faz necessário a análise de todos os rios (Silva, 2012).

$D d=\frac{L}{A}$

O Quadro 2 apresenta a classificação da densidade de drenagem.

Quadro 2: Classificação da densidade de drenagem.

\begin{tabular}{|c|c|}
\hline $\mathrm{DD} \leq 0,5 \mathrm{KM} / \mathrm{KM}^{2}$ & DRENAGEM POBRE \\
\hline $0,5 \leq \mathrm{DD}<1,5 \mathrm{KM} / \mathrm{KM}^{2}$ & DRENAGEM REGULAR \\
\hline $1,5 \leq \mathrm{DD}<2,5 \mathrm{KM} / \mathrm{KM}^{2}$ & DRENAGEM BOA \\
\hline $2,5 \leq \mathrm{DD}<3,5 \mathrm{KM} / \mathrm{KM}^{2}$ & DRENAGEM MUITO BOA \\
\hline
\end{tabular}

Fonte: Feitosa et al., (2011).

\subsubsection{Aspectos referentes ao relevo}

Para Elebson et al. (2011) estudar os aspectos referentes ao relevo define precisamente o caminho em que o escoamento da agua superficial perfaz observado no mundo real. Logo, esse tópico trata das metodologias utilizadas com o relevo para as análises hidrológicas.

\subsubsection{Declividade média da microbacia}

O relevo tem ligação direta com o potencial do escoamento, infiltração e topografia da bacia (Vitte \& Vilela Filho, 2006). Destaca-se a Declividade, no qual é uma razão direta entre o produto do somatório das curvas de níveis e a distância entre as curvas pela área (Equação 4).

$$
\begin{aligned}
& S=\left(\frac{D * L}{A}\right) * 100 \\
& \text { Onde: } \\
& \mathrm{S}=\text { declividade média }(\%) \\
& \mathrm{D}=\text { distância entre as curvas de nível }(\mathrm{km})
\end{aligned}
$$


$\mathrm{L}=$ comprimento total das curvas de nível $(\mathrm{km})$

$\mathrm{A}=$ área da bacia hidrográfica $\left(\mathrm{km}^{2}\right)$

Sendo assim o Quadro 3 apresenta a classificação quanto à declividade.

Quadro 3: Classificação quanto à declividade.

\begin{tabular}{|c|c|}
\hline DECLIVIDADE (\%) & CLASSIFICAÇÃO \\
\hline $0-3$ & RELEVO PLANO \\
\hline $3-8$ & Relevo suave ondulado \\
\hline $8-20$ & RELEVO ONDULADO \\
\hline $20-45$ & RELEVO FORTE ONDULADO \\
\hline $45-75$ & RELEVO MONTANHOSO \\
\hline$>75$ & RELEVO ESCARPADO \\
\hline
\end{tabular}

Fonte: EMBRAPA (1979).

\subsection{Análise pluviométrica}

Segundo Villela e Mattos (1975), a avaliação do comportamento das chuvas é necessária para uso frequente em aplicação nos projetos hidráulicos. Para definição do posto pluviométrico com maior influência na microbacia em estudo, analisou-se visualmente os postos através do site da Fundação Cearense de Meteorologia e Recursos Hídricos - FUNCEME (http://www.funceme.br/), no qual foi apresentado a localização e os dados de todos os postos pluviométricos do estado do Ceará.

Também, analisou-se além de proximidade, através de auxílio do software Excel, se os dados do posto em estudo possuem uma série climatológica de grande tamanho, apresentando, quando houver períodos sem dados.

Essa definição ocorre de forma simplificada devido ao tamanho da bacia hidrográfica em estudo e o distanciamento em os postos pluviométricos na área de interesse.

\subsubsection{Determinação do preenchimento de falhas}

A partir da definição dos postos utilizados no estudo, foi realizado o preenchimento das possíveis falhas, através do método de regressão linear. Esse método estima os coeficientes da equação linear, no qual envolve uma ou mais variáveis independentes que melhor preveem o valor da variável dependente. De acordo com Tucci (2001), segue os seguintes procedimentos:

- Por meio do Excel, destaca-se todas as falhas, presentes no período de dados definido para ser utilizado, no posto em utilização;

- É selecionado os dados de datas próximas as falhas do posto, por exemplo, um mês antes e um depois de cada falha;

- Assim da mesma maneira, seleciona-se os dados do mesmo período de datas de um posto próximo ao de estudo;

- Aplicados todos os dados em colunas e ordenados por data, é realizado uma seleção dos mesmos, apagando-se os dados de dias em que a altura pluviométrica de ambos seja igual a 0 (zero);

- Por fim é gerado um gráfico de dispersão com os dados em conformidade, de forma que Y seja o posto que se deseja preencher, e X, o posto utilizado para o preenchimento;

- A partir do gráfico, é traçado uma linha de tendência linear entre os dados, e gerado uma equação que a represente, assim como o valor do coeficiente de determinação $\left(\mathrm{R}^{2}\right)$, que mostra o ajuste da equação;

- A partir da equação de regressão dos dados, preenche-se as falhas presentes no posto, levando em consideração o valor que o posto escolhido apresenta na mesma data da falha.

\subsubsection{Análise de consistência dos dados}

Após o preenchimento dos dados, se faz necessário "analisar o grau de homogeneidade dos dados disponíveis no posto, em relação ao registrado no posto vizinho" (Tucci, 2001, p. 186). Assim foi realizada a soma mensal, acumulando a 
cada mês do período do posto em análise. A partir desses dados, plota-se um gráfico de dispersão com X sendo o posto analisado e $\mathrm{Y}$ do posto vizinho, onde metodologicamente seria necessário o uso da média dos três postos com maior influência, porém para esse estudo utilizou-se de um posto, pela alta proximidade e a grande similaridade dos dados, mantendo em destaque os meses em que houve falhas preenchidas.

Através da linearidade observada entre os pontos do gráfico e a relativa proximidade a 1 (um) do $R^{2}$ da análise de correlação, pode-se determinar se os dados dos postos apresentam consistência, assegurando assim a homogeneidade dos dados.

\subsubsection{Distribuição de frequência}

Para Villela e Mattos (1975), a previsão de chuvas em uma determinada região se dá por meio analítico dos dados acumulados, resultantes de eventos já ocorridos, em que se observa a frequência através da série total registrada, em proporção anual, mensal ou em um período qualquer definido na análise realizada. É possível a elaboração gráfica da frequência das precipitações ocorridas, mas para isso é necessário a identificação das máximas precipitações ocorridas na região da bacia hidrográfica, em que após a devida identificação desses dados realiza-se a divisão desses dados em intervalos variáveis, no qual é possível obter a frequência da ocorrência dessa 'precipitação dentro desse intervalo predefinido na análise. Por meio do método Califórnia, classifica-se os valores dos dados em ordem decrescente, em que na sequência faz-se o uso da Equação 5.

$F=\frac{m}{n}$

Sendo (m) a ordem da classificação da precipitação, e (n) o período em anos contido na análise. É necessário entender que quanto maior o número de intervalos analisados, mais precisos e abrangentes serão os resultados, pois o intervalo será definido a partir do quantitativo de chuvas em $\mathrm{mm} / \mathrm{h}$, determinados no início do estudo, com base no objetivo da pesquisa, onde nesse estudo adotou-se intervalo de chuvas de $25 \mathrm{~mm}$. Através disso passa a ser possível a determinação do número de classes das chuvas ocorridas, no qual se dá por intermédio dos intervalos determinados no estudo, entre a menor e a maior precipitação ocorrida na bacia.

Assim, é preciso analisar o modelo que melhor se adeque ao tipo de distribuição e variáveis contidas na amostra. Conforme Naghettini e Pinto (2007) o modelo de distribuição gama é o que melhor se adequa a realidade desse estudo, visto que tal distribuição é feita com base na mediana dos dados, possibilitando a analise em períodos diversos. Portanto, é necessária uma verificação para identificar se o modelo escolhido estava em conformidade ao modelo probabilístico, sendo esse o teste do Qui-quadrado, no qual pode ser utilizada a Equação 6.

$X^{2}=\sum_{I=1}^{K} \frac{(0 i-E i)^{2}}{E i}$

Sendo (k) os possíveis resultados, (Ei) a ocorrência dos resultados e (Oi) os resultados que se espera. Segundo Naghettini e Pinto (2007) a resposta obtida por meio da equação anteriormente citada, é verificado em comparação aos dados contidos em uma tabela referência, no qual é relacionado a um grau de liberdade, e um respectivo intervalo de confiança. Em que o devido grau de liberdade é calculado como (k-1) em que o k é a quantidade de classes, e o intervalo de confiança nos mostra o quanto uma amostra é tendenciosa a retornar resultados previstos. 


\subsubsection{Teste Qui-quadrado}

De acordo com Sharpe (2015), o teste do qui-quadrado é utilizado com finalidade de conferir se a frequência absoluta de uma determinada variável é expressivamente distante da distribuição de frequência absoluta esperada. Ou seja, esse teste verifica a adequabilidade de um modelo probabilístico a um conjunto de dados de um experimento (Singh et al., 2011).

Palomino et al. (2009) complementam ao afirmar que há vários tipos de testes qui-quadrado, como o de qualidade de ajuste, bastante utilizado para verificar a qualidade do ajuste de uma amostra de dados categóricos a uma distribuição teórica, como também o de associação e independência, utilizados para verificar a associação para determinar se uma variável está conexa a outra variável; e para verificar se o valor encontrado de uma variável é dependente de outra variável, respectivamente.

\subsection{Tempo de concentração}

De acordo com Queiroz e Alves (2020), é a duração em que o fluido fica retido na superfície. Para sua determinação foi utilizada a equação de Kirpich (Equação 7), no qual L é a extensão do curso d'água em km e S a declividade entre a cabeceira do talvegue principal até o exutório em metros.

$$
t c=0,019 * \frac{4^{0,787}}{[S)^{0,395}}
$$

\subsection{Intensidade Pluviométrica e Chuva de Projeto}

Bodas Terassi et al. (2018) afirmam que a determinação da intensidade através do método racional é uma razão entre o tempo e o espaço. O método racional relaciona a chuva com o escoamento superficial (Coletto \& Santos, 2018), no qual considera o tempo de duração da chuva semelhante ao período de concentração da bacia. Segundo Lou (2010), a utilização do método racional é o mais habitual em análises de bacias menores que $0,8 \mathrm{~km}^{2}$, por ser de fácil compreensão e fornecer bons resultados.

Esse método relaciona a precipitação com o deflúvio, considerando as principais características da bacia, tais como área e o coeficiente de escoamento superficial, em que a vazão de dimensionamento é calculada pela Equação 8.

$$
Q=C * I * A
$$

Onde Q= Vazão em $\left(\mathrm{m}^{3} / \mathrm{s}\right) ; \mathrm{C}=$ Coeficiente de Runnof; I= Intensidade pluviométrica em $(\mathrm{mm} / \mathrm{h}) ; \mathrm{A}=$ Área da bacia em $\left(\mathrm{m}^{2}\right)$. Os coeficientes de escoamento superficial são adotados em função do tipo e uso do solo, (SEIBT et al., 2012), como pode ser verificado no Quadro 4.

Quadro 4: Coeficientes de escoamento superficial de alguns materiais.

\begin{tabular}{|c|c|c|}
\hline Superfícies & Intervalo & $\begin{array}{c}\text { C } \\
\text { valor esperado }\end{array}$ \\
\hline Cimento e asfalto & $0,90-0,95$ & 0,95 \\
\hline Paralelepípedo & $0,58-0,81$ & 0,60 \\
\hline Blockets de concreto & $0,70-0,89$ & 0,78 \\
\hline Concreto e asfalto poroso & 0,05 & 0,03 \\
\hline Solo compactado & $0,59-0,79$ & 0,66 \\
\hline
\end{tabular}

Fonte: Pereira Júnior (2020). 
Lou (2010) utilizou esse modelo hidrológico com finalidade de realizar uma simulação na Sub Bacia da cidade de Pedro do Rio Estado do Rio de Janeiro (RJ). Como resultado, o autor verificou que o Método Racional possuiu tendência de superestimar vazões de cheia, tendo em vista que ele é mais adequado para bacias de menor porte do que as sub-bacias simuladas no estudo.

Assim, para uso e aplicação do método racional, a intensidade de chuva utilizada é a máxima contida em determinado intervalo de tempo com um tempo de recorrência definido. No entanto, para esse método o intervalo de tempo será considerado o mesmo do tempo de concentração da bacia. Logo, foi calculado a intensidade de chuva para o município do Barro em um tempo de retorno de 48 anos, justificando-se esse período pelo fato de conhecer a intensidade ocorrida na maior precipitação sobre a microbacia que tem um período probabilístico de retorno a cada 48 anos, fazendo uso da Equação que Batista (2018) define na (Equação 9):

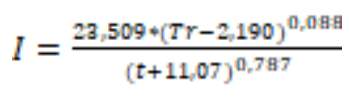

Logo para determinação da intensidade da chuva de projeto, fez-se o uso da Equação 6 citada anteriormente, utilizando uma Tr tempo de retorno para 5 anos. O tempo (t) em minutos considerado para obtenção dos resultados foi o mesmo encontrado para o tempo de concentração (Tc), como nos indica o modelo utilizado para obtenção dos resultados dessa pesquisa que é o método racional.

\subsection{Vazão máxima e vazão de projeto}

Baseado no método racional faz-se a utilização da Equação 8, que é indicada para análise em pequenas bacias, por demandar poucos dados e ser de fácil solução, para que a vazão fosse calculada tomou por base os dados da intensidade pluviométrica, encontrada com base na Equação 9. Para o cálculo da vazão de projeto adota-se o mesmo procedimento, com base no valor encontrado da chuva de projeto. A Equação 10 foi utilizada para determinação das vazões.

$$
Q=\frac{C * \pi * A}{2 \cdot 6}
$$

Onde:

C: coeficiente de perdas (Runnof);

I: intensidade da precipitação em $\mathrm{mm} / \mathrm{min}$;

A: área da bacia em $\mathrm{km}^{2}$;

Q: vazão em m³/s;

\section{Resultados e Discussões}

No processo de delimitação da microbacia hidrográfica urbana, obteve-se a microbacia apresentada na Figura 2. 
Figura 2: Imagem da microbacia hidrográfica e seu exutório.

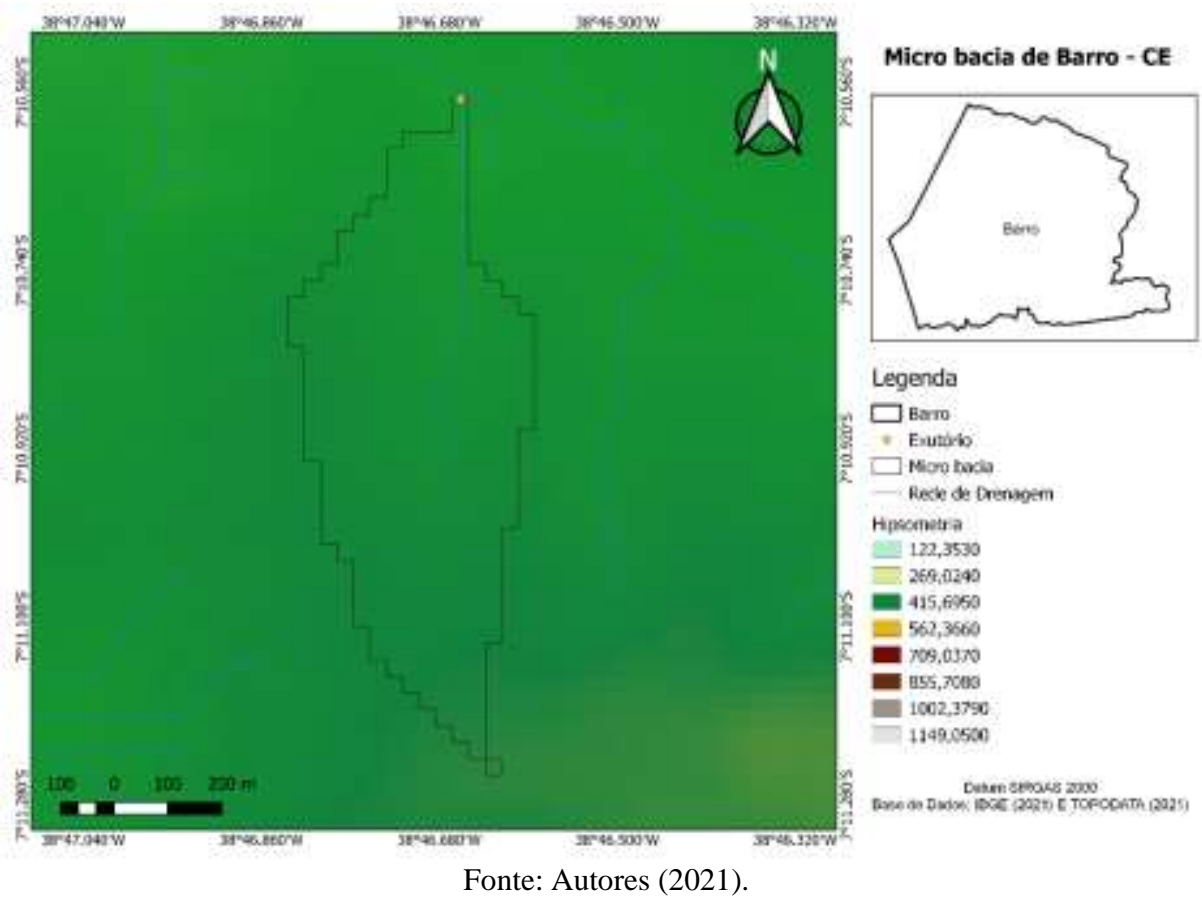

A partir da delimitação da bacia e do uso do software QGIS, foi possível obter os dados do Quadro 5, no qual serão utilizados na análise morfométrica.

Quadro 5: Dados da microbacia

\begin{tabular}{|c|c|c|c|}
\hline Dados (QGIS) & Unidade & Abreviação & Valor \\
\hline Área de drenagem & $\mathrm{km}^{2}$ & $\mathrm{~A}$ & 0,32 \\
\hline Perímetro de drenagem & $\mathrm{km}$ & $\mathrm{P}$ & 3,5 \\
\hline Comprimento axial & $\mathrm{km}$ & $\mathrm{L}$ & 1,26 \\
\hline Comprimento das curvas de nível & $\mathrm{km}$ & $\mathrm{Ln}$ & 3,37 \\
\hline Comprimento dos cursos d'água & $\mathrm{km}$ & $\mathrm{Lc}$ & 0,86 \\
\hline Comprimento do rio principal & $\mathrm{km}$ & $\mathrm{Lrp}$ & 0,86 \\
\hline
\end{tabular}

Fonte: Autores (2021).

A relação dos dados contidos na Tabela 5 são de extrema importância para que a morfometria da bacia possa ser definida, sendo possível observar que se trata de uma microbacia urbana de pequena extensão, com um único curso d'agua, sendo esse o curso principal.

Através da obtenção dos resultados da Tabela 6, e por meio das equações apresentadas no item 4.2 foi possível realizar a análise dos parâmetros morfométricos da microbacia, apresentados no Quadro 6. 
Quadro 6: Parâmetros morfométricos da microbacia em estudo.

\begin{tabular}{|c|c|c|c|}
\hline Parâmetros morfométricos & Unidade & Abreviação & Valor \\
\hline Coeficiente de compacidade & - & $\mathrm{Kc}$ & 1,23 \\
\hline Fator de forma & - & $\mathrm{Kf}$ & 0,20 \\
\hline Declividade média da bacia & $\%$ & $\mathrm{Dm}$ & 5,27 \\
\hline Densidade de drenagem & $\mathrm{km} / \mathrm{km}^{2}$ & $\mathrm{Dd}$ & 2,69 \\
\hline
\end{tabular}

Fonte: Autores (2021).

A partir da análise da Tabela 6, verificou-se que através de uma análise quanto a forma da microbacia, o seu coeficiente de compacidade ( $\mathrm{Kc}$ ) foi de 1,23, no qual é possível afirmar que a microbacia possui um formato alongado, tendo uma menor possibilidade de acontecer enchentes. Logo, obtendo a confirmação da informação por meio do fator de forma (Kf), no qual o seu valor é de 0,20 sendo menor que 0,5, que de acordo com Goehr et al. (2015), a bacia possui menores tendências a enchentes.

Além disso, a declividade apresentou um valor de 5,27 \%, sendo classificada como microbacia em uma região de relevo suave ondulado, de acordo com Embrapa (1979), tendo característica de microbacia de baixa velocidade, consequentemente gerando um escoamento superficial de menor velocidade, resultando em maior tempo para que a água alcance o exutório. Já na drenagem da microbacia, foi possível observar que apresenta uma densidade de drenagem de 2,69 $\mathrm{km} / \mathrm{km}^{2}$, que segundo Feitosa, Santos e Araújo (2011), indica uma drenagem muito boa.

Para análise da precipitação na microbacia, foi adotado a utilização do posto pluviométrico mais próximo à microbacia, no qual o posto 21, da cidade de Barro - CE, foi o posto utilizado para esta análise. Os dados do referido posto estão contidos no Quadro 7.

Quadro 7: Dados do posto pluviométrico utilizado no estudo.

\begin{tabular}{|c|c|c|c|c|c|c|}
\hline Município & Posto & Latitude & Longitude & Ano Inicial & $\begin{array}{c}\text { Ano } \\
\text { Final }\end{array}$ & Falhas \\
\hline Barro & 21 & 7,183861 & 38,777361 & 1974 & 2020 & $\begin{array}{c}28 / 01 / 2007 \mathrm{e} \\
27 / 10 / 2010\end{array}$ \\
\hline
\end{tabular}

Fonte: Funceme (2021). 
Figura 3: Dados da precipitação máxima anual posto Barro

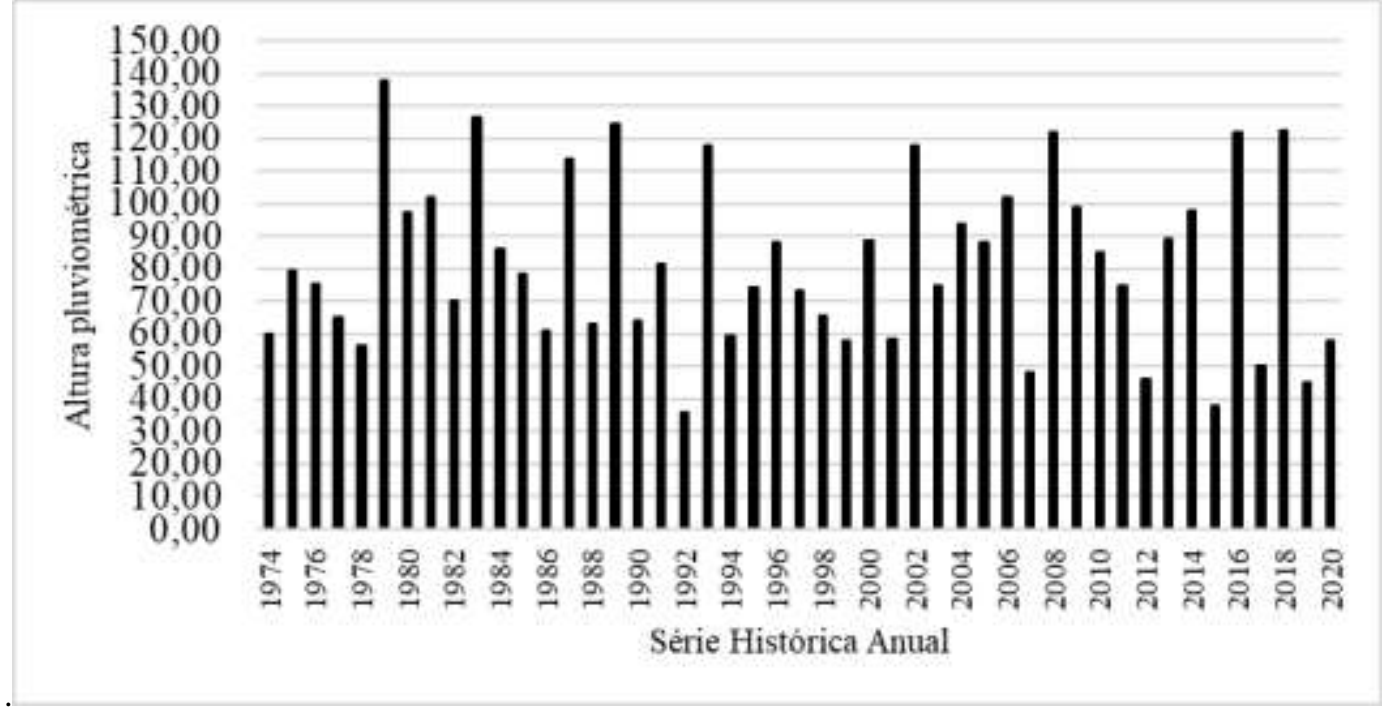

Fonte: FUNCEME (2021).

Para o preenchimento das falhas do posto em análise, tomou por base o posto 87 da cidade de Mauriti-CE, visto que, através de análise feita no site da FUNCEME, esse foi o posto com maior índice de dados coletados e menor índice de falhas, próximo ao posto em análise. Sendo também de igual período, com relação ao posto utilizado para determinação das precipitações na região. O processo de preenchimento de falhas contidas nas datas de 28/01/2007 e 27/10/2010 do posto 21 Barro - CE, foram realizadas com base nos dados do posto 87 Mauriti - CE, no qual apresentou uma equação de regressão linear com $\mathrm{R}^{2}$ aceitável (Figura 4).

Figura 4: Regressão linear (relação entre os dados pluviométrico dos postos Barro e Mauriti).

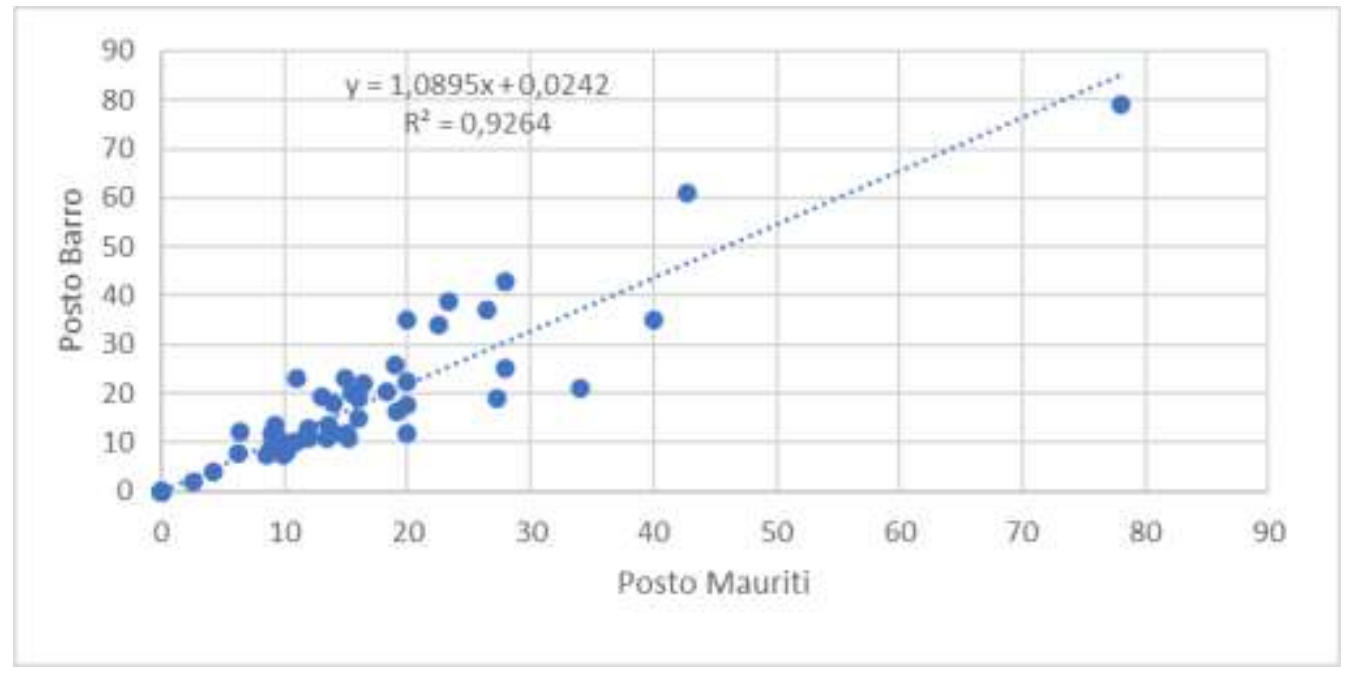

Fonte: Autores (2021).

Com o devido preenchimento feito, foi realizado a análise de consistência entre os dados existentes e os preenchidos por meio da relação entre a precipitação mensal acumulada do posto 21 (Barro) e a precipitação mensal acumulado do posto 87 (Mauriti), assim tendo como satisfatório o resultado obtido na Figura 5. 
Figura 5: Análise de consistência de dados dos postos de Barro x Mauriti nas séries de 1974-2020.

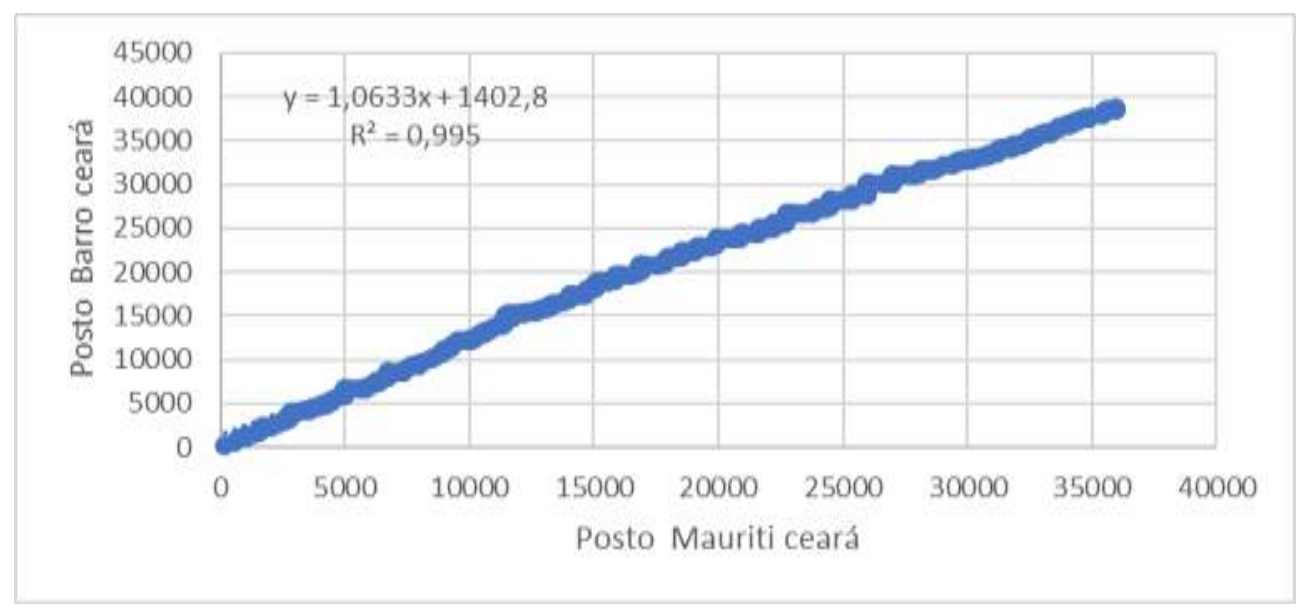

Fonte: Autores (2021).

Após análise feita com base nas chuvas máximas anuais diária, foi possível compreender que a maior chuva anual diária ocorrida no intervalo de 47 anos na região da microbacia em estudo foi 137,6 mm/dia (Figura 6). Esse dado é muito importante pois através do mesmo é possível determinar a intensidade de chuva e a vazão máxima, como também o comportamento pluviométrico da região em um intervalo de tempo.

Neste ponto, foi realizado a divisão das precipitações máximas em que o intervalo pré-definido foi de $25 \mathrm{~mm}$ de chuva, no qual obteve-se a amplitude de 7 classes, na ordem de $0 \mathrm{~mm}$ como limite inferior e $175 \mathrm{~mm}$ como limite superior. Sendo o Fi equivalente ao número de vezes em que as precipitações máximas se repetem dentro do intervalo determinado. E o Fe que tem como base de cálculo a distribuição Gama, que para isso faz-se necessário o uso dos dados obtidos no Quadro 8, como também do teste de qui-quadrado apresentado no Quadro 9.

Quadro 8: Coeficientes.

\begin{tabular}{|c|c|}
\hline \multicolumn{2}{|c|}{ Coeficientes Gama - Cálculo } \\
\hline $\mathrm{A}$ & 0,05 \\
\hline$\alpha$ & 9,19 \\
\hline$\beta$ & 8,83 \\
\hline$\Gamma(\alpha)$ & 60408,63 \\
\hline
\end{tabular}

Fonte: Autores (2021). 
Quadro 9: Distribuição gama.

\begin{tabular}{|c|c|c|c|c|c|c|c|c|c|c|}
\hline \multicolumn{10}{|c|}{ TESTE DE QUI-QUADRADO } \\
\hline $\begin{array}{c}\mathbf{N}^{\mathbf{0}} \text { da } \\
\text { Classe }\end{array}$ & $\begin{array}{c}\text { Limite } \\
\text { Inferior }\end{array}$ & $\begin{array}{c}\text { Limite } \\
\text { Superior }\end{array}$ & $\mathbf{f i}$ & $\mathbf{x i}$ & $\mathbf{f i . x i}$ & $\mathbf{l n}(\mathbf{x})$ & $\mathbf{l n}(\mathbf{x}) . \mathbf{f i}$ & $\begin{array}{c}\text { Dist. Gama } \\
\text { Acum. }\end{array}$ & $\mathbf{F e}$ & $\chi^{\mathbf{2}}$ \\
\hline 1 & 0 & 25 & $\mathbf{0}$ & 12,5 & 0 & $2,53 \mathrm{E}+00$ & $0,00 \mathrm{E}+00$ & $2,07 \mathrm{E}-03$ & $\mathbf{0}$ & 0,097 \\
\hline 2 & 25 & 50 & $\mathbf{5}$ & 37,5 & 187,5 & $3,62 \mathrm{E}+00$ & $1,81 \mathrm{E}+01$ & $1,07 \mathrm{E}-01$ & $\mathbf{5}$ & 0,001 \\
\hline 3 & 50 & 75 & $\mathbf{1 5}$ & 62,5 & 937,5 & $4,14 \mathrm{E}+00$ & $6,20 \mathrm{E}+01$ & $4,51 \mathrm{E}-01$ & $\mathbf{1 6}$ & 0,084 \\
\hline 4 & 75 & 100 & $\mathbf{1 6}$ & 87,5 & 1400 & $4,47 \mathrm{E}+00$ & $7,15 \mathrm{E}+01$ & $7,78 \mathrm{E}-01$ & $\mathbf{1 5}$ & 0,025 \\
\hline 5 & 100 & 125 & $\mathbf{9}$ & 112,5 & 1012,5 & $4,72 \mathrm{E}+00$ & $4,25 \mathrm{E}+01$ & $9,36 \mathrm{E}-01$ & $\mathbf{7}$ & 0,344 \\
\hline 6 & 125 & 150 & $\mathbf{2}$ & 137,5 & 275 & $4,92 \mathrm{E}+00$ & $9,85 \mathrm{E}+00$ & $9,85 \mathrm{E}-01$ & $\mathbf{2}$ & 0,050 \\
\hline 7 & 150 & 175 & $\mathbf{0}$ & 162,5 & 0 & $5,09 \mathrm{E}+00$ & $0,00 \mathrm{E}+00$ & $9,97 \mathrm{E}-01$ & $\mathbf{1}$ & 0,558 \\
\hline$\Sigma$ & & & $\mathbf{4 7}$ & & $\mathbf{3 8 1 2 , 5}$ & $\mathbf{2 9 , 4 9 4 1 3}$ & $\mathbf{2 0 4 , 0 4 9 2 5 1}$ & & & $\mathbf{1 , 1 6}$ \\
\hline
\end{tabular}

Fonte: Autores (2021).

Assim, é possível observar conforme Quadro 10 o grau de liberdade obtido, o intervalo de confiança e o Quiquadrado tabelado. Conforme Pearson e Hartley (1996), o modelo de distribuição tem compatibilidade com a análise realizada.

Quadro 10: Estatística.

\begin{tabular}{|l|l|c|c|}
\hline Menor Precipitação & 36 & Grau de Liberdade & 6 \\
\hline Maior Precipitação & 137,6 & Intervalo de Confiança & $95 \%$ \\
\hline $\mathrm{N}^{\circ}$ de Amostras & 47 & Qui-quadrado (tabelado) $\left(\chi^{2} .95\right)$ & 12,6 \\
\hline Amplitude & 7 & - & - \\
\hline
\end{tabular}

Fonte: Autors (2021).

A Figura 6 apresenta a distribuição de frequência, na qual tem como referência a distribuição de frequências das chuvas observadas versos a distribuição de frequência esperada.

Figura 6: Distribuição de frequência

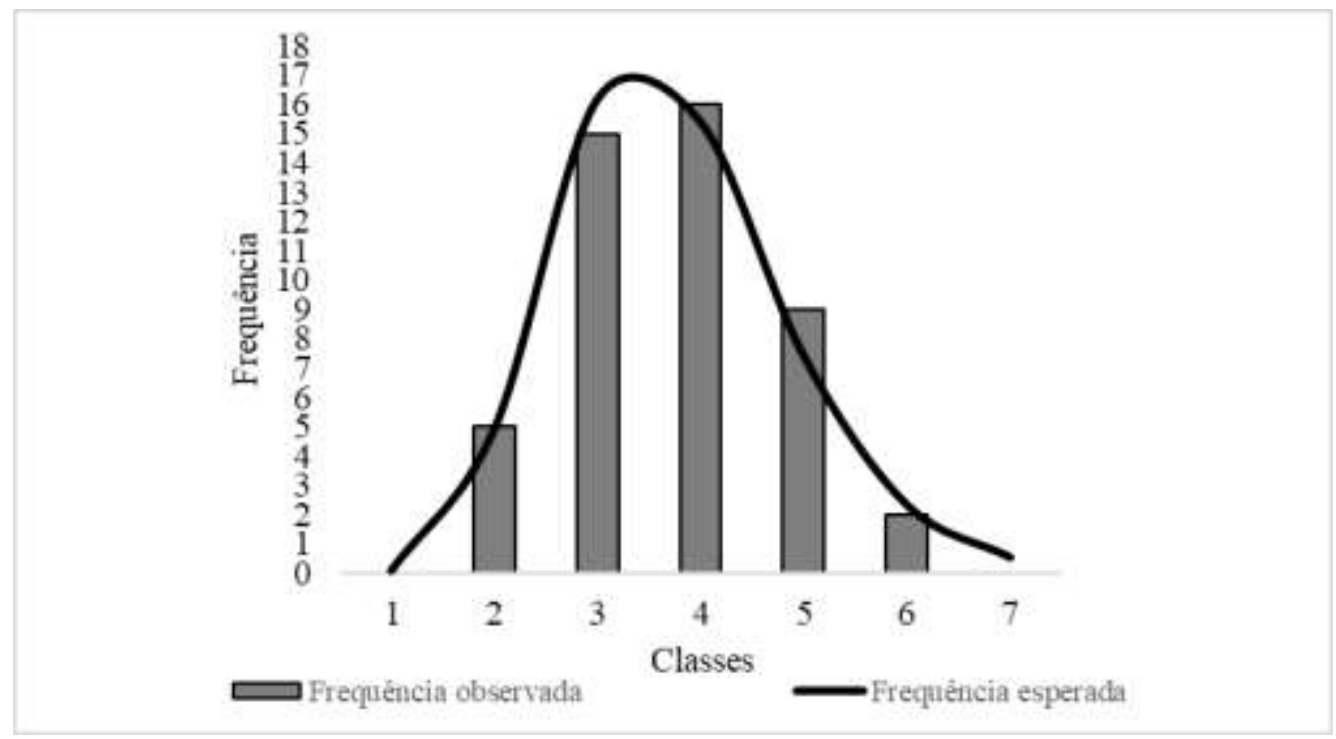

Fonte: Autors (2021).

Para o modelo utilizado, o tempo de concentração é altamente importante, visto que, é utilizado também para determinação da intensidade de chuva. Logo, o tempo de concentração (Tc) determinado para essa microbacia foi de 10,41 
minutos, porém, de acordo com Teixeira (2010), a equação de Kirpich utilizada, tende a minorar o tempo de concentração, consequentemente influenciando na determinação da intensidade de chuva e elevando a vazão máxima.

A intensidade pluviométrica foi determinada com base na Equação 9, tendo como resultado uma intensidade pluviométrica de 2,95 $\mathrm{mm} / \mathrm{min}$ referente a um tempo de retorno de 48 anos, que, comparado com os resultados encontrados na determinação da chuva máxima média anual, é superior, demonstrando uma majoração no resultado obtido. Sendo assim, determinou-se a intensidade de chuva de projeto no valor de $2,30 \mathrm{~mm} / \mathrm{min}$ para um período de retorno de 5 anos, que comparando as médias anuais diárias contidas no posto de maior influência sobre a bacia, encontrou-se precipitações de igual intensidade demonstrando coerência nos dados obtidos, em que para caso de dimensionamento hidráulico, essa seria a informação utilizada para determinação da vazão de projeto.

Em um estudo sobre a viabilidade para implantação de Biovaletas no sistema de drenagem de Porto Velho - RO, Neves Moura et al. (2017) identificaram uma intensidade pluviométrica inferior ao resultado encontrado nesse estudo (49,51 $\mathrm{mm} / \mathrm{h})$ ou $(0,825 \mathrm{~mm} / \mathrm{min})$, representando $0,29 \%$ das precipitações registradas entre 2006 - 2014. Essa diferença é devido a diferença de pluviosidade das duas regiões, como também os métodos utilizados nos diferentes estudos não foram os mesmos.

Na determinação da vazão pluviométrica que se deu com base na Equação 10, utiliza-se o valor encontrado da intensidade pluviométrica tendo como resultado uma vazão (Q) de $2,44 \mathrm{~m} 3 / \mathrm{s}$, no qual se caracteriza uma vazão considerável para a microbacia em estudo, em seguida, também foi possível determinar a vazão de projeto baseado no valor da intensidade de projeto encontrada, resultando em uma vazão de projeto $(\mathrm{Q})$ de $2,04 \mathrm{~m} 3 / \mathrm{s}$, considerada também uma vazão considerável. Porém, Lou (2010) afirma que o método racional tende a majorar o cálculo da vazão.

Resultados semelhantes foram encontrados nos estudos de Seibt et al (2012) na bacia hidrográfica do córrego Ruivinho - GO. A microbacia hidrográfica localiza-se no município de Goiânia e apresenta uma extensão de 1,37km, e área de $0,94 \mathrm{~km}^{2}$ e declividade média de $5 \%$. Os autores obtiveram uma vazão de $16,25 \mathrm{~m}^{3}$, um valor superior ao encontrado na presente pesquisa, em que se justifica pela diferença climatológica da região.

\section{Considerações Finais}

Com base nos dados obtidos, foi possível realizar a caracterização morfométrica da microbacia, no qual pode-se caracterizar como alongada e de baixa tendência a enchentes, e com uma inclinação referente a relevo ondulado suave, com boa densidade de drenagem, resultando assim, em uma menor velocidade no escoamento, apesar de praticamente não existir sinuosidade. Analisando a pluviometria, verificou-se que a região é de chuvas periódicas, e que sinaliza uma grande predominância de chuvas máximas entre 75 e 100 mm, e uma mínima recorrência de chuva entre 125 e 150mm, de acordo com o tempo de retorno encontrado para cada média estatística realizada e a análise de distribuição de frequência.

A conclusão desse estudo vem a confirmar problemas de drenagem em época chuvosa, porém, a bacia apresenta pouca tendência a enchentes. Mostrando que o problema ocasionado na região em estudo, não vem por meio da caracterização da microbacia e sim pelo uso inadequado do solo da região, por meio de atividade antrópica não planejada, acarretando consequências ao leito da microbacia, como também, pela ausência de um sistema de drenagem no local, fazendo com que toda água precipitada sobre a microbacia escoe de forma superficial, sendo esses os principais motivos causadores desse problema na região.

\section{Referências}

Alves, G. J. (2016). Aplicabilidade do Método CN-SCS a uma bacia hidrográfica representativa dos latossolos no Sul de MG. 156 f. Dissertação de Mestrado em Recursos Hídricos em Sistemas Agrícolas - Universidade Federal de Lavras.

Araújo, L. E. et al. (2009). Bacias hidrográficas e impactos ambientais. Qualitas Revista Eletrônica, 8(1). 
Barboza, E. N. et al. (2020). Influência da arborização nas variáveis climáticas em ruas com e sem asfaltamento na cidade de Barbalha-CE. Brazilian Journal of Development, 6(1), 980-986.

Batista, T. L. (2018). Geração de equações IDF dos municípios cearenses pelo método de desagregação por isozonas implementado em um programa computacional. 96 f. Dissertação de Mestrado - Universidade Federal do Ceará.

Bispo, T. C., \& Levino, N. A. (2011). Impactos ambientais decorrentes do uso e ocupação desordenada do solo: um estudo da região da periferia de Maceió/AL. In: Encontro Nacional De Engenharia De Produção, 31, 2011, Belo Horizonte. Anais eletrônicos... ABEPRO.

Bodas Terassi, P. M. et al. (2018). Frequência e Intensidade Pluviométrica na Região Norte do Estado do Paraná, Brasil. Revista do Departamento de Geografia, 35, 121-133.

Carvalho, D. F. de., \& Silva, L. D. B. da. (2006). Escoamento superficial. Universidade Federal Rural do Rio de Janeiro.

Coletto, L. A., \& Dos Santos, A. T. (2018). Análise das vazões máximas na área urbana de São Miguel do Oeste por meio do método racional e comparação com valores observados em campo. Unoesc \& Ciência-ACET, 9(1), 15-22.

Costa Filho, E. (2017). Determinação Do Hidrograma Unitário Instantâneo Geomorfológico Para A Bacia Do Rio Beberibe, Pernambuco, Brasil. 2017. 88 f. Trabalho de Conclusão de Curso - Escola Politécnica de Pernambuco - Universidade de Pernambuco.

Cunha, Z. A., Beskow, S., \& Moura, M. M de. (2017). Aplicabilidade do método número da curva (CN/SCS) com base em valores de CN tabelados: estudo de caso na bacia hidrográfica do arroio cadeia. In: Simpósio Brasileiro de Recursos Hídricos, 22(1), 1-8.

Danciguer, G. M., \& Reis, E. A. (2017). Prado dos. Pavimento Permeável Aplicado em Área Urbana, como Medida de Escoamento da Água da Chuva. ETICEncontro De Iniciação Científica. 13(13).

Elesbon, A. A. A. et al. (2011). Uso de dados SRTM e plataforma SIG na caracterização morfométrica da bacia hidrográfica do Braço Norte do Rio São Mateus-Brasil. Rem: Revista Escola de Minas, 64(3), 281-288.

EMBRAPA. Classes de declividade (EMBRAPA, 1979). https://ceivap.org.br/sesmarias/MAPA-SESMARIA-EMBRAPA-90-60.pdf

Feitosa, A., Santos, B. O., \& Araújo, M. do S. B. (2011). Caracterização morfométrica e identificação de áreas susceptíveis a erosão na bacia do rio Pajeú, PE: o estudo de caso da bacia do rio Pajeú-PE. Revista Brasileira de Geografia Física, 4(4), 820-836.

FUCEME. Portal hidrológico. http://www.hidro.ce.gov.br/

Garcez, L. N., \& Alvarez, G. A. (1988). Hidrologia. Editora Blucher.

Goehr, A. P. L. D. et al. (2015). Caracterização morfológica e hidrológica da microbacia hidrográfica bálsamo, Campo Grande.

INnocente, C., \& Chaffe, P. L. B. (2017). Uma revisão preliminar sobre a aplicação do hidrograma unitário na pesquisa, no ensino e na engenharia. In: XXII Simpósio Brasileiro de Recursos Hídricos, 22(1), 1-8.

IPECE. Perfil básico municipal Barro. https://www.ipece.ce.gov.br/wp-content/uploads/sites/45/2018/09/Barro_2006.pdf

Justino, E. A., Paula, H. M., \& Paiva, E. d. C. R. (2011). Análise do efeito da impermeabilização dos solos urbanos na drenagem de água pluvial do município de Uberlândia-MG. Espaço em revista, 13(2).

Lima, N. A. (2014). Comparação entre métodos de dimensionamento de sistemas de drenagem em aeródromos. 2014.143 f. Trabalho de Conclusão de Curso - Instituto Tecnológico de Aeronáutica.

Lins, R. C. (2019). Modelagem hidráulica do canal Beira Rio nos cenários de requalificação do curso d'água e de implantação de bacia de detenção. 2019. 65 f. Dissertação (Mestrado em Engenharia Civil) - Universidade Federal de Pernambuco.

Lou, R. F. (2010). Modelagem hidrológica chuva-vazão e hidrodinâmica aplicada na bacia experimental do rio Piabanha/RJ. 190 f. Dissertação de Mestrado (Programa de Pós-graduação em Engenharia Civil) - Universidade Federal do Rio de Janeiro, 2010.

Maia, A. L., Amaral, I. R., \& Versiani, B. R. (2006). Metodologia DPFT de identificação do Hidrograma Unitário e das Precipitações Efetivas: Estudo de caso para a bacia hidrográfica de Juatuba-MG. Revista Brasileira de Recursos Hídricos, 11(1), 79-90.

MAPBIOMAS. Uso e cobertura do solo: Estatística de cobertura. https://plataforma.brasil.mapbiomas.org/.

Marinho Filho, G. M. et al. (2013). Modelos hidrológicos: conceitos e aplicabilidades. Revista de Ciências Ambientais, 6(2), 35-47.

Martins, C. M. T. et al. (2010). Hidrologia urbana: conceitos básicos. Série Cursos Técnicos, n. 1.

Maus, V. W., Righes, A. A., \& Buriol, G. A. (2007). Pavimentos permeáveis e escoamento superficial da água em áreas urbanas. I Simpósio De Recursos Hídricos Do Norte E Centrooeste, v. 1.

Naghettini, M., \& Pinto, É. J. de A. (2007). Hidrologia estatística. CPRM.

Neves Moura, M. et al. (2017). Estudo De Viabilidade Para Implantação De Biovaletas No Sistema De Drenagem Urbana De Porto Velho-RO. In: Simpósio Brasileiro de Recursos Hídricos, 20(1), 1-7. 
Palomino, M. A., Oakes, M. P., \& Wuytack, T. (2009). Automatic extraction of keywords for a multimedia search engine using the chi-square test. In: Proceedings of the 9th Dutch-Belgian information retrieval workshop, 3-10.

Pearson, E. S., \& Hartley, H. O. (1996). Biometrika tables for statisticians. In: AGRIS, 1(1).

Pereira Júnior, A. M. M. (2020). Pavimentação por bloco intertravado de concreto de vias públicas. file:///C:/Users/Downloads/20201007083724_MES_DES_ESTACIONAMENTO_R00.pdf

Queiroz, M. S., \& De Sousa Alves, N. (2020). Aplicação De Diferentes Fórmulas De Tempo De Concentração Para Uma Bacia Hidrográfica Urbana. Revista Tocantinense de Geografia, 9(18), 219-231.

Rodrigues, V. A. et al. (2015). Avaliação do escoamento e interceptação da água das chuvas. Irriga, 1(1), 01-13.

Seibt, A. C. et al. (2012). Comparação das vazões de projeto pelo método racional e método tempo área para bacias urbanas do município de GoiâniaGO. REEC-Revista Eletrônica de Engenharia Civil, 4(2).

Sharpe, D. (2015). Chi-square test is statistically significant: Now what? Practical Assessment, Research, and Evaluation, $20(1), 8$.

Silva, K. A. da. (2007). Análise da eficiência de métodos de controle de Enchentes na atenuação de picos de cheias utilizando o modelo computacional SWMM Storm Water managemente model. 2007. 125 f. Dissertação de Mestrado - Universidade Federal de Goiás.

Silva, L. P. E. (2007). Modelagem e geoprocessamento na identificação de áreas com risco de inundação e erosão na bacia do rio Cuiá. $2007.118 \mathrm{f}$. Dissertação de mestrado em Engenharia Urbana - Universidade Federal da Paraíba.

Silva, Q. D. (2012). Mapeamento geomorfológico da Ilha do Maranhão. 2012. Tese de Doutorado - Universidade Estadual de São Paulo.

Singh, P. K., Maehiwal, D., \& Roy, M. K. (2011). Modeling daily runoff and probabilistic estimation of design maximum daily runoff from selected watersheds of Udaipur, Rajasthan. Indian Journal of Soil Conservation, 39(3), 176-182.

Souza, S. R., \& Sousa, E. O. (2020). Potabilidade da água de cacimbas: estudo de caso em um distrito da cidade de Barro, Ceará. Revista Brasileira de Engenharia de Biossistemas, 14(4), 321-328.

Targa, M. dos S. et al. (2012). Urbanização e escoamento superficial na bacia hidrográfica do Igarapé Tucunduba, Belém, PA, Brasil. Revista Ambiente \& Água, 7(2), 120-142.

Tasca, F. A. et al. (2017). O papel da drenagem urbana na prevenção de desastres hidrológicos na Bacia hidrográfica do rio Itajaã Açu. Revista brasileira de cartografia, 69(1), $129-142$.

Teixeira, C. A. Apostila de Hidrologia Aplicada. https://engenhariacivilfsp.files.wordpress.com/2014/03/hidro_celimar.pdf

Teodoro, V. L. I. et al. (2007). O conceito de bacia hidrográfica e a importância da caracterização morfométrica para o entendimento da dinâmica ambiental local. Revista Brasileira Multidisciplinar, 11(1), 137-156.

Tonello, K. C. et al. (2006). Morfometria da bacia hidrográfica da Cachoeira das Pombas, Guanhães-MG. Revista Árvore, 30(5), 849-857.

Torrico, J. J. T. (1974). Práticas hidrológicas. Transcon.

Tucci, C. E. M. (2001). Hidrologia Ciência e Aplicação. (2a ed.), Editora da UFRGS.

Tucci, C. E. M. (1993). Água no meio urbano. Livro água doce, 1-40.

Tucci, C. E. M. (2003). Drenagem urbana. Ciência e cultura, 55(4), 36-37.

Vitte, A. C., \& Vilela Filho, L. R. (2006). A urbanização, a fragilidade potencial do relevo e a produção do risco na bacia hidrográfica do Córrego Proença, município de Campinas, Brasil. Territorium, 13, 105-114.

Zanandrea, F. (2016). Avaliação de técnicas de baixo impacto no controle de impactos hidrológicos em uma bacia urbana em consolidação. $2016.93 \mathrm{f}$. Dissertação de Mestrado - Universidade Federal do Rio Grande do Sul. 